\title{
Invited Response on: Letter to the Editor on "Extracellular Vesicles From a Three-Dimensional Culture of Perivascular Cells Accelerate Skin Wound healing in a Rat"
}

\author{
Jong Hun Lee ${ }^{1,2}$
}

Received: 21 April 2021 / Accepted: 22 April 2021 / Published online: 11 May 2021

(C) Springer Science+Business Media, LLC, part of Springer Nature and International Society of Aesthetic Plastic Surgery 2021

Level of Evidence $V$ This journal requires that authors assign a level of evidence to each article. For a full description of these Evidence-Based Medicine ratings, please refer to the Table of Contents or the online Instructions to Authors www.springer.com/00266.

To the Editor,

We would like to thank you for your interest in our research and article and for some questions and comments through the Letter to the Editor. We would like to respond to them.

Firstly, we applied a mixture of EV and hydrogel inside the full thickness skin defect wound and dressed using Tegaderm (3M, St. Paul, MN, USA) it. Additionally, we used male Sprague-Dowley rats (Crl:CD(SD), 16 wks) in our study, and did not measure their weight, but most of them were about the same size.

Secondly, we measured the positive area as image $\mathrm{J}$ on the tissue slide and compared the differences between groups by displaying the results on Fig 4, 6, and 8. In addition, we have confirmed the expression of CD31 and
VEGF stimulating angiogenesis, which are mainly used for evaluating angiogenesis, and thank you for your recommendation.

Finally, further research will be conducted on how EVs separated from 3D-cultured PVCs have a better effect on wound healing than PVC-2D-EVs.

\section{Declarations}

Conflicts of interest The authors declare that they have no conflicts of interest to disclose.

Human and animal rights All of procedures to animal were performed after obtaining approval from Eulji University Institutional Animal Care and Use Committee (EUIACUC-16-20). All experiments were performed following the approved protocol in accordance with the institutional guidelines.

Informed consent Informed consent does not apply to this type of animal study.

Publisher's Note Springer Nature remains neutral with regard to jurisdictional claims in published maps and institutional affiliations.
Jong Hun Lee

joaljh@eulji.ac.kr

1 Department of Plastic and Reconstructive Surgery, Nowon Eulji Medical Center, School of Medicine, Eulji University, 68, Hangeulbiseok-ro, Nowon-gu, Seoul, Republic of Korea

2 Eulji Medi-Bio Research Institute, Eulji University, Seoul, Republic of Korea 